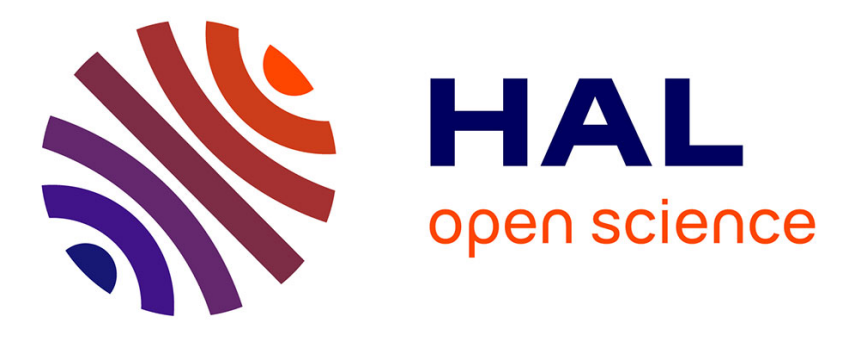

\title{
Graph-based analysis of brain connectivity during spelling task
}

Mahmoud Hassan, Ahmad Mheich, Fabrice Wendling, Olivier Dufor, Claude Berrou

\section{- To cite this version:}

Mahmoud Hassan, Ahmad Mheich, Fabrice Wendling, Olivier Dufor, Claude Berrou. Graph-based analysis of brain connectivity during spelling task. ICABME 2013 : 2nd International Conference on Advances in Biomedical Engineering, Sep 2013, Tripoli, Lebanon. pp.191-194. hal-00949906

\section{HAL Id: hal-00949906 https://hal.science/hal-00949906}

Submitted on 15 Apr 2014

HAL is a multi-disciplinary open access archive for the deposit and dissemination of scientific research documents, whether they are published or not. The documents may come from teaching and research institutions in France or abroad, or from public or private research centers.
L'archive ouverte pluridisciplinaire HAL, est destinée au dépôt et à la diffusion de documents scientifiques de niveau recherche, publiés ou non, émanant des établissements d'enseignement et de recherche français ou étrangers, des laboratoires publics ou privés. 


\section{Graph-based analysis of brain connectivity during spelling task}

\author{
Mahmoud Hassan, Ahmad Mheich, Fabrice Wendling \\ Laboratoire Traitement du Signal et de L'Image, \\ INSERM, University of Rennes 1 \\ Rennes, France \\ firstname.name@univ-rennes1.fr
}

\author{
Olivier Dufor, Claude Berrou \\ Télecom Bretagne (Institut Mines-Télécom) \\ UMR CNRS Lab-STICC \\ Brest, France \\ firstname.name@telecom-bretagne.eu
}

\begin{abstract}
Most of the brain functions are based on interactions between neuronal assemblies distributed within and across distinct cerebral regions. A major challenge in neuroscience is to identify these networks from neuroimaging data. In this paper, we investigate the brain connectivity during a specific cognitive task: spelling the name of an object represented on a picture. By using high-resolution electroencephalography (hr-EEG) and phase synchrony analysis combined with graph theory-based analysis, we show that the topographic distribution of the phase synchrony and the graph parameters are very powerful tools to disclose the activated macro-regions involved in such a complex task.
\end{abstract}

Keywords_High resolution EEG; Evoked potentials; Brain connectivity; Cognitive analysis; Graph theory.

\section{INTRODUCTION}

Brain connectivity is at the heart of many studies in neurosciences, either in the field of cognitive [1] or clinical [2] research. The main idea is that networks of interconnected distinct brain regions underlie normal brain functions (involved in learning, memory, behaviour adaptation to stimuli, emotions) as well as pathological processes in some brain disorders like epilepsy, autism or schizophrenia. Generally speaking, the terminology "brain connectivity" refers to three different concepts: i) "structural connectivity" which denotes anatomical links, ii) "functional connectivity" which rely on statistical dependencies between signals from different brain areas and iii) "effective connectivity" which introduces causal interactions among these signals [3].

Recently, the quantitative description of the topological organization of brain networks based on graph theory has received an increasing interest [4-6]. An original theory socalled the "mental information theory" was developed by Berrou et al. with the aim of designing new neuro-inspired intelligent machines $[7,8]$.

This work has received a French government support granted to the CominLabs excellence laboratory and managed by the National Research Agency in the "Investing for the Future" program under reference ANR-10LABX-07-01.

This work is supported and partly granted by the Committee for Clinical Research (COREC) at the CHU of Rennes.
Briefly, it considers any cortical network as an informational model of recurrent graph where each node is not a single neuron, but a group of neurons, called a microcolumn. These microcolumns can merge to form columns (clusters of neurons) that in turn, form macrocolumns together.

In this model, mental pieces of information, called "infons", are carried by cliques, that is, small combinations of microcolumns that activate together. The clique can be viewed as a sub-graph in the vast network of microcolumns present in the human neocortex.

By introducing some properties of redundant coding and parcimony in the recurrent graph model, authors showed theoretically how neural cliques are forming during a cognitive process.

Further to the theory, we assume that the activation of brain networks at the "micro-level" i.e. clique will lead to the activation of brain networks at the "macro-level" i.e. the brain surface. Thus, the purpose of the present paper is to detect the activated "macro-networks" using hr-EEG recorded during a spelling task.

\section{MATERIAL AND METHODS}

\section{A. EEG recordings and pre-processing}

Nine subjects were shown pictures $(\mathrm{n}=148)$ and were asked to spell the name of the object displayed on them. The 148 images are selected from the database of 400 pictures standardized for French [9] and is divided into two sessions (eight minutes each) of 74 stimuli.

The brain activity was acquired using high resolution EEG recording system (hr-EEG, EGI Electrical Geodesic Inc.) allowing for the positioning of 256 electrodes on the head of the participant. The unique feature of this system is the "almost full" coverage of the subject's head by surface electrodes. This increased number of electrodes offers the possibility to better estimate the overall activity of the neocortex from non-invasive scalp measurements. Moreover, the temporal resolution of the recorded signals is excellent (in the order of $1 \mathrm{~ms}$ ) as EEG signals are collected with a $1 \mathrm{kHz}$ sampling frequency and filtered at $3-45 \mathrm{~Hz}$.

An averaging process is done on stimulus-aligned epoch of $920 \mathrm{~ms}$ starting from the moment the picture appeared on 
screen (200 ms are added to the analysis as pre-stimulus considered as baseline).

Each trial was visually inspected, and epochs contaminated by eye blinking, movements or other noise were rejected and excluded from the analysis.

\section{B. Phase synchony analysis}

Several methods have been proposed to analyse the statistical coupling between signals. These methods can be categorized in three families: linear and nonlinear regression, phase synchronisation and general synchronisation (see [10] for review). As a first step and given that we are dealing with short signal (600 to $900 \mathrm{~ms}$ ), a good candidate method appears to be the phase synchronisation (PS) method which has been widely used in the field of ERPs brain connectivity [11]. The general principle of the PS method is to detect the existence of a phase locking between two systems defined as:

$$
\varphi(t, n)=\left|\Phi_{x}(t, n)-\Phi_{y}(t, n)\right| \leq C
$$

where $\Phi_{x}(t ; n), \Phi_{y}(t, n)$ are the unwrapped phases of the signals $(x$ and $y)$ representative of the two systems at time bins $t$, trial $n[1, \ldots, N]$ and $C$ a constant. The first step for estimating the phase synchronization is to extract the instantaneous phase of each signal. Two different techniques can be used: the Hilbert transform and the wavelet transform. In this paper we are using the method based on Hilbert transform as it has recently been shown that the application of both approaches (i.e., HT and wavelet transform) produces essentially the same result [12].

The second step is the definition of an appropriate index to measure the degree of synchronization between estimated instantaneous phases. We are using the phase locking value (PLV) defined as:

$$
P L V_{t}=\frac{1}{N}\left|\sum_{n=1}^{N} e^{i \varphi(t, n)}\right|
$$

PLV is a normalized measure varying between 0: random phase difference and 1: constant phase difference.

To illustrate the connectivity in predefined time windows, the average PLVs in each window are computed.

\section{Graph analysis}

Using a graph theory based analysis, we extracted parameters from the connectivity graphs computed using the PLV in order to characterize these graphs at the different instant (from the Onset to the motor response). Each electrode is considered as a node and the significant PLVs are representing the edges. In this paper we focus on the difference between left and right hemisphere. The main parameters extracted from both sides are the following:

- The degree: The number of links connected to a node.

- The Number of Edges (NE): The number of links in the network.
- The Betweenness Centrality (BC): The ratio between the number of shortest paths passing through a specific node and the total number of shortest paths in the network.

- The density (D): The fraction of present connections to all possible connections of a network.

- The lateralization Index (LI): compute the difference between left and right hemisphere and defined as:

$$
L I(B C)=\left(\frac{B C(\text { Right })-B C(\text { Left })}{B C(\text { Right })+B C(\text { Left })}\right) * 100
$$

with LI being negative ( -100 to 0$)$ or positive ( 0 to 100$)$ when $\mathrm{BC}$ is more prominent over the left hemisphere or the right hemisphere respectively. Note that we are only considering the values intra-hemisphere while the inter-hemisphere connections are ignored.

The analyzed graphs here are considered as unweighted and undirected. For more details about these parameters and others, see $[4,13]$.

\section{RESULTS}

\section{A. ERP analysis}

The Fig. 1 shows the ERPs averaged over all subjects and trials. The signal length is chosen as the minimal value of all the reaction times for the sessions. We could clearly delineate several potentials like the positivity 150 (P150) which reflects the activity in early visual extrastriate cortex that is involved in the initial phase of visual feature processing and which is sensitive to changes in those elementary features whatever the object is: a picture [14] or a letter drawing [15]. In accordance with a large number of behavioral studies, using pictures and words, we also detected a negativity around $200 \mathrm{~ms}$ which reflects the access to the semantic of the picture; a process known to take place before the phonological one [16].

Finally, we also observed the P3a et P3b potentials whose properties reveal the link between the intentional system and the ongoing process [17].

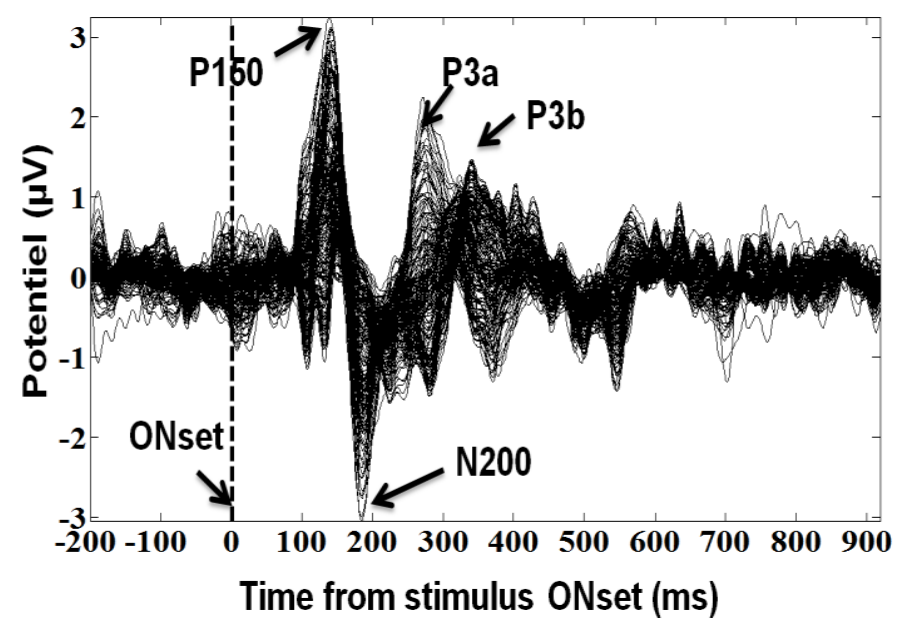

Figure1: The average ERP signals over subjects and trials. 

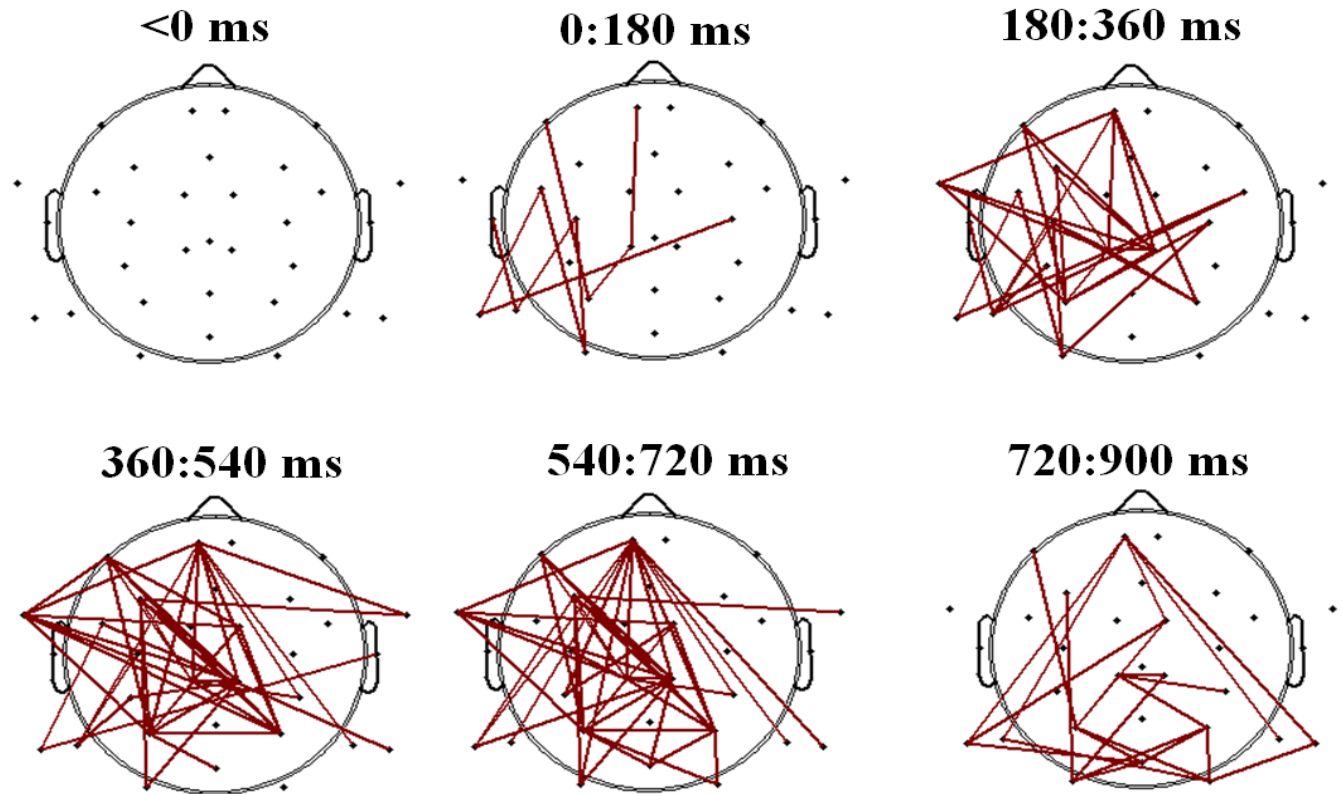

Figure 2 Topographic distributions of the phase locking values (PLV) during the spelling task for a representative subject ( 0 represent the stimulus instant).

\section{B. Brain connectivity}

An example of the spatiotemporal distribution of phase synchrony from $200 \mathrm{~ms}$ before the stimulation onset $(0 \mathrm{~ms})$ to motor response $(>720 \mathrm{~ms})$ is presented in Figure 2. A classical symmetric montage of 32 electrodes is used for visualization simplicity. The lines represent the significant phase synchronization values between pairs of electrodes (compared to shuffled data with $p<0.01$, see [11]).

It can be observed that, immediately after the onsets, within a period that grossly correspond to the visual processing (0$180 \mathrm{~ms}$ ), synchronies are present in left parieto-occipital and fronto-temporal regions. The graph parameters $\mathrm{BC}, \mathrm{NE}$ and $\mathrm{D}$ (summarized in table I) are clearly higher in the left side of the brain with $0.022,8$ and 0.087 against 0,0 and 0 respectively. Consequently, the lateral index LI equals to -100 (full left lateralization).

A remarkable increase in the phase synchrony is then observed during the next $180 \mathrm{~ms}(180-360 \mathrm{~ms})$ and located essentially at the left occipital, frontal and temporal regions which are supposed to be involved at this stage while recovering the names of the images [18]. BC, NE and D $(0.12$, 16 and 0.175$)$ at the left and $(0,0$ and 0$)$ at the right with $\mathrm{LI}=$ 100 confirm this lateralization. Also the degrees of the different node show that the channel at the left central parietal, left frontal parietal and left temporal (and frontal temporal) have the highest degrees (number of connected links) which reflect the channels (and the regions) of interest supposed to be active during this task stage.

The phase synchronization between $360 \mathrm{~ms}$ and $720 \mathrm{~ms}$ present a more spatial distribution of the connectivity in both hemispheres with always a superiority of the left side ( $\mathrm{BC}=0.06, \quad \mathrm{NE}=18$ and $\mathrm{D}=0.197)$ ) on the right side $(\mathrm{BC}=0.003, \mathrm{NE}=3$ and $\mathrm{D}=0.03)$ with $\mathrm{LI}=-88.5$.
Finally, a phase desynchronization occurs and a diminution of every indices is observed at the temporal and parietal sites while the motor response is given $(720-900 \mathrm{~ms})$ (left: $\mathrm{BC}=0.001, \mathrm{NE}=5, \mathrm{D}=0.05$ and right: $\mathrm{BC}=0.0009, \mathrm{NE}=2$ and $\mathrm{D}=0.02$ ) with $\mathrm{LI}=-33.3$ ).

These results corroborate those reported in the neuroimaging literature, as processes implicated in the spelling network are predominantly located in the left hemisphere [18, 19]. However, ones need to delineate more "cognitively" the different period of time the brain goes through.

\section{Discussions}

Preliminary results were presented to demonstrate that hr-EEG can be used to reveal the cortical network(s) involved in a specific cognitive task. These observations are promising regarding the accordance with the state of the art on language processing and the complexity of the task which, among several processes, involves the working memory system at stake in the mental information theory [5-6].

The connectivity graphs presented here showed the brain networks behavior at different stage: from visual processing to motor response. The time window used for computing the graph of brain connectivity is chosen based on the state of the art regarding similar cognitive task. We are working on more optimal choice by using a method referred to a spatiotemporal segmentation of the ERPs [20].

We highlight that the results do not represent the "cliques" as they are defined in the mental information theory. However, we consider these results as an excellent preliminary step.

Our ongoing work is focusing on analyzing the network model of brain connectivity referred to the graph theory based analysis at the source level by passing from electrode level to the "source" level using inverse methods (MUSIC) to get closer to "neural cliques". 
Table I: Graph parameters extracted from left and right hemisphere

\begin{tabular}{|c|c|c|c|c|c|c|}
\cline { 2 - 7 } \multicolumn{1}{c|}{} & & $0: 180 \mathrm{~ms}$ & $180: 360 \mathrm{~ms}$ & $360: 540 \mathrm{~ms}$ & $540: 720 \mathrm{~ms}$ & $720: 900 \mathrm{~ms}$ \\
\hline \multirow{4}{*}{ Left } & $\mathrm{BC}$ & 0.022 & 0.12 & 0.107 & 0.06 & 0.001 \\
\cline { 2 - 7 } & $\mathrm{NE}$ & 8 & 16 & 18 & 18 & 5 \\
\cline { 2 - 7 } & $\mathrm{D}$ & 0.0879 & 0.175 & 0.197 & 0.197 & 0.05 \\
\hline \multirow{3}{*}{ Right } & $\mathrm{BC}$ & 0 & 0 & 0 & 0.037 & 0.0009 \\
\cline { 2 - 7 } & $\mathrm{NE}$ & 0 & 0 & 1 & 3 & 2 \\
\cline { 2 - 7 } & $\mathrm{D}$ & 0 & 0 & 0.011 & 0.033 & 0.022 \\
\hline
\end{tabular}

More graph parameters will be explored specially those offering information about the presence of sub-networks inside the whole complex network, such as the "modularity" and "community" parameters.

\section{CONCLUSION}

In this paper, we presented a study aiming to identify the cortical regions involved during a spelling task. The use of the hr-EEG and the connectivity analysis (phase locking value) indicated clearly that the left hemisphere is much more implicated during the spelling at the different instant than the right hemisphere. The basic graph parameters (betweenness centrality, density, number of edges and the lateralization index) have showed high capacity to quantify this lateralization.

\section{REFERENCES}

[1] M. C. Stevens, "The developmental cognitive neuroscience of functional connectivity," Brain and cognition, vol. 70, pp. 1-12, 2009.

[2] P. J. Uhlhaas and W. Singer, "Neural synchrony in brain disorders: relevance for cognitive dysfunctions and pathophysiology," Neuron, vol. 52, pp. 155-168, 2006.

[3] O. Sporns, Networks of the Brain: MIT Press, 2010.

[4] M. Rubinov and O. Sporns, "Complex network measures of brain connectivity: uses and interpretations," Neuroimage, vol. 52, pp. 1059-1069, 2010.

[5] P. Hagmann, L. Cammoun, X. Gigandet, R. Meuli, C. J. Honey, V. J. Wedeen, and O. Sporns, "Mapping the structural core of human cerebral cortex," PLoS biology, vol. 6, p. e159, 2008.

[6] D. Meunier, R. Lambiotte, A. Fornito, K. D. Ersche, and E. T. Bullmore, "Hierarchical modularity in human brain functional networks," Frontiers in neuroinformatics, vol. 3, 2009.

[7] C. Berrou, "Graphs, codes and the brain," in Communications, Networking and Applications for the Internet of Things, Wireless Personnal Multimedia Communications (WPMC), Brest, France, 2011.

[8] C. Berrou and V. Gripon, Petite mathématique du cerveau. Une théorie de l'information mentale. Paris: Odile Jacob 2012.

[9] F. X. Alario and L. Ferrand, "A set of 400 pictures standardized for French: Norms for name agreement, image agreement, familiarity, visual complexity, image variability, and age of acquisition," Behavior Research Methods, vol. 31, pp. 531-552, 1999.
[10] F. Wendling, K. Ansari-Asl, F. Bartolomei, and L. Senhadji, "From EEG signals to brain connectivity: a model-based evaluation of interdependence measures," Journal of neuroscience methods, vol. 183, pp. 918,2009 .

[11] E. Rodriguez, N. George, J. P. Lachaux, J. Martinerie, B. Renault, and F. J. Varela, "Perception's shadow: long-distance synchronization of human brain activity," Nature, vol. 397, pp. 430-433, 1999.

[12] R. Quian Quiroga, A. Kraskov, T. Kreuz, and P. Grassberger, "Performance of different synchronization measures in real data: a case study on electroencephalographic signals," Phys Rev E Stat Nonlin Soft Matter Phys, vol. 65, 2002.

[13] L. d. F. Costa, F. A. Rodrigues, G. Travieso, and P. Villas Boas, "Characterization of complex networks: A survey of measurements," Advances in Physics, vol. 56, pp. 167-242, 2007.

[14] M. Eddy, A. Schmid, and P. J. Holcomb, "Masked repetition priming and event - related brain potentials: A new approach for tracking the time - course of object perception," Psychophysiology, vol. 43, pp. 564-568, 2006.

[15] J. P. Petit, J. K. MidglEy, J. P. Holcomb, and J. Grainger, "On the time course of letter perception: A masked priming ERP investigation," Psychonomic Bulletin \& Review, vol. 13, pp. 674-681, 2006.

[16] B. M. Schmitt, A. Rodriguez-Fornells, M. Kutas, and T. F. Münte, "Electrophysiological estimates of semantic and syntactic information access during tacit picture naming and listening to words," Neuroscience Research, vol. 41, pp. 293-298, 2001.

[17] M. D. Comerchero and J. Polich, "P3a and P3b from typical auditory and visual stimuli," Clinical Neurophysiology, vol. 110, pp. 24-30, 1999

[18] C. Price, C. Moore, G. Humphreys, R. Frackowiak, and K. Friston, "The neural regions sustaining object recognition and naming," Proceedings of the Royal Society of London. Series B: Biological Sciences, vol. 263, pp. 1501-1507, 1996.

[19] J. J. Purcell, P. E. Turkeltaub, G. F. Eden, and B. Rapp, "Examining the central and peripheral processes of written word production through meta-analysis," Frontiers in psychology, vol. 2, 2011.

[20] C. Perret and M. Laganaro, "Comparison of electrophysiological correlates of writing and speaking: A topographic ERP analysis," Brain topography, vol. 25, pp. 64-72, 2012. 